Pathologe 2020 41 (Suppl 2):S96-S98 https://doi.org/10.1007/s00292-020-00882-w Online publiziert: 11 . Dezember 2020 (c) Springer Medizin Verlag GmbH, ein Teil von Springer Nature 2020

\author{
P. Boor ${ }^{1} \cdot$ A. Hartmann ${ }^{2}$ \\ ${ }^{1}$ Institut für Pathologie \& Medizinische Klinik II (Nephrologie), Sektion Translationale Nephropathologie, \\ Universitätsklinikum der RWTH Aachen, Aachen, Deutschland \\ ${ }^{2}$ Institut für Pathologie, Universitätsklinikum Erlangen, Erlangen, Deutschland
}

\title{
Bericht zur Late-Breaking Session Pathologie und COVID-19
}

lichkeits- und Pressearbeit der Deutschen Gesellschaft für Pathologie (DGP), des Bundesverbandes Deutscher Pathologen (BDP) und einiger KollegInnen hervorgehoben, die eine bemerkenswerte Resonanz in der Gesellschaft gefunden hat ein für unser Fach und die Wahrnehmung von Autopsien sehr wichtiger Aspekt.

Nach der Einleitung folgten zwei Beiträge von Herrn Prof. Boor. Im ersten Vortrag, wurde das weltweit erste nationale Register für COVID-19 Obduktionen vorgestellt - das DeRegCOVID [1] (www.DeRegCOVID.ukaachen.de). Es wurde in enger Unterstützung mit der DGP und dem BDP aufgebaut und verfolgt folgende Ziele:

1. zentrale elektronische Erfassung möglichst aller COVID-19 Obduktionen in Deutschland;

2. Unterstützung alle Zentren u. a. durch Bereitstellung von Empfehlungen und Verfahrensanweisungen für COVID-19 Obduktionen;

3. Unterstützung von Wissenschaftlern bei adäquater Nutzung von Autopsieproben;

4. Vermittlung zwischen Wissenschaftlern und Zentren und

5. zentrale Melde- und Datenauswertungsstelle.

Dabei verfolgt das Register folgende grundsätzliche Prinzipien. Alle Proben bleiben dezentral und durchgehend im Besitz der jeweiligen Zentren und diese behalten auch die Rechte über die jeweiligen Daten. In dem Vortrag wurden die Details der Prozesse und Abläufe sowie die gesammelten Daten in dem Register beschrieben sowie auch Beispiele wie das Register Forschungsprojekte erleichtern kann. Das DeRegCOVID wird durch das Bundesministerium für Gesundheit unterstützt (ZMVI1-2520COR201).

Im zweiten Vortrag von Herrn Prof. Boor wurden die SARS-CoV-2 Nachweismethoden in pathologischem Material, insbesondere durch Autopsien, diskutiert. Es wurden die Vorteile und mögliche Nachteile von RNA-basierten Methoden aufgeführt, vor allem der klassischen Abstriche aus dem NaseRachen-Bereich, der Lunge oder der Hornhaut (letztere ist bei Hornhauttransplantationen relevant), des PCRbasierten Nachweises aus FFPE Material sowie auch die FFPE-basierten FISH und CISH Methoden. Es wurden Beispiele von in situ-Hybridisierungen gezeigt (FISH und CISH), die eine genaue Lokalisation des Virus, z.B. in Endothelzellen, ermöglichen. Zusätzlich wurde kurz die Problematik der immunhistochemischen und ultrastrukturellen elektronenmikroskopischen Nachweismethoden diskutiert und insgesamt die Empfehlung formuliert, sich auf die RNA-basierten Methoden, und insbesondere die PCR-basierte Methode aus FFPE, zu fokusieren. Diese Methoden werden auch am Universitätsklinikum Aachen angeboten.

Den dritten Beitrag hielt Herr Prof. Danny Jonigk aus dem Institut für $\mathrm{Pa}$ thologie der Medizinischen Hochschule Hannover, der über seine herausragende Studie zur histopathologischen, ultrastrukturellen und molekularen Charakteristika des pulmonalen Befalles bei COVID-19 berichtete. In dieser Studie wurde das COVID-19-Muster mit normalen Lungen sowie Lungen von an akutem Atemnotsyndrom (ARDS) verstor- 
benen Patienten als Folge einer Influenza $\mathrm{A}(\mathrm{H} 1 \mathrm{~N} 1)$-Infektion verglichen. Es konnte gezeigt werden, dass das führende histomorphologische Bild eines diffusen Alveolarschadens wie bei anderen ARDS prinzipiell auch bei COVID-19 besteht, zusätzlich aber signifikant häufiger als bei HiN1 weitere spezifische Veränderungen auftreten, insbesondere eine deutliche Endothelialschädigung sowie eine vermehrt vorkommende Mikroangiopathie mit Mikrothromben und intussuszeptiver Angiogenese. Obgleich diese Studie nur auf einer kleinen Fallserie beruht, wurde sie in der renommierten Zeitschrift New England Journal of Medicine publiziert und bietet den Grundsatz für das Verständnis der führenden und fatalen pulmonalen Manifestationen von COVID-19 [2].

Frau Prof. Zsuzsanna Varga und Herr Prof. Holger Moch aus Zürich berichteten über ihre Arbeiten zu Endothelzellinfektion und Endothelialitis bei Covid-19 (siehe Beitrag in diesem Verhandlungsband). Sie konnten in Obduktionsmaterial sowohl durch In-situ-Hybridisierung als auch durch Elektronenmikroskopie das Virus in kleineren Gefäßen nachweisen und in verschiedenen Organen eine eindeutige Endothelialitis zeigen [3]. Die direkte virale Infektion der Endothelzellen waren mit einer ausgeprägten endothelialen Entzündung assoziiert. Diese Arbeiten wurden in Lancet veröffentlicht. Auch die Züricher Kollegen konnten zahlreiche leukozytäre Thromben in verschiedenen Organen nachweisen. Diese Daten zeigen die Entwicklung einer Endothelialitis mit vaskulärer Konstriktion und konsekutiver Organischämie, Entzündung und Gewebeschädigung sowie mikrothrombotischen $\mathrm{Mi}$ krozirkulationsstörung und erklären das höhere Risiko eines schweren Verlaufs der COVID-19-Erkrankung bei Patienten mit kardiovaskulären Erkrankungen und kardiovaskulären Risikofaktoren.

Frau Prof. Karin Klingel aus Tübingen sprach über die Evidenz der Mitbeteiligung des Herzens bei Covid-19-Infektion. Sie konnte zeigen, dass eine direkte Infektion des Myokards selten stattfindet. Zusätzlich stellte Sie die Schwierigkeiten des Nachweises der Viruspartikel in der Elektronenmikroskopie dar.

Pathologe 2020 - 41 (Suppl 2):S96-S98 https://doi.org/10.1007/s00292-020-00882-w

(c) Springer Medizin Verlag GmbH, ein Teil von Springer Nature 2020

\section{P. Boor · A. Hartmann}

\section{Bericht zur Late-Breaking Session Pathologie und COVID-19}

\section{Zusammenfassung}

Der vorliegende Beitrag fast kurz die Late-Breaking Session "Pathologie und COVID-19", die im Rahmen der virtuellen Jahrestagung der deutschen Gesellschaft für Pathologie am 06. Juni 2020 stattgefunden hat, zusammen. Die Beiträge informierten über das Deutsche Register für COVID19 Obduktionen (DeRegCOVID), über die Nachweismethoden von SARS-CoV-2 in pathologischem Material, über die typischen Lungenbefunde bei schweren COVID-19Fällen, über die deutlichen (mikro)vaskulären Veränderungen sowie die kardialen und gastrointestinalen Befunde bei COVID-19Erkrankten. Zusammenfassend wurde in dieser ersten allgemeinen wissenschaftlichen Sitzung in der deutschen Pathologie zur COVID-19-Pandemie deutlich, dass die PathologInnen in Deutschland, Österreich und der Schweiz sehr schnell auf die Pandemie reagiert und an zahlreichen Stellen ein Autopsieprogramm aufgebaut haben, das zu medizinisch hoch-relevanten Befunden geführt hat.

\section{Schlüsselwörter}

SARS-CoV-2 · Detektionsmethoden · Diffuser Alveolarschaden · Thromben · AutopsieRegister

\section{Late Breaking Session Pathology and COVID-19 Report}

\section{Abstract}

This paper briefly summarizes the latebreaking session "Pathology and COVID-19" that took place at the virtual congress of the German Society of Pathology on June 6, 2020. The lectures tackled a broad variety of aspects, including the German Registry for COVID19 autopsies (DeRegCOVID), the detection methods of SARS-CoV-2 in pathological material, the typical lung findings in severe COVID-19 cases, the distinct (micro)vascular changes and the cardiac and gastrointestinal involvement in COVID-19. In summary, in this first scientific meeting in German pathology on the COVID-19 pandemic, it became clear that pathologists in Germany, Austria and Switzerland have reacted very quickly to the pandemic and have established an autopsy program that has led to medically highly relevant findings.

Keywords

SARS-CoV-2 - Detection methods · Diffuse alveolar damage $\cdot$ Thrombi $\cdot$ Autopsy registry
Zum Abschluss der Session stellte Frau Dr. Christina Falkeis-Veits aus Bayreuth die Rolle von Covid-19 bei gastrointestinalen Entzündungen dar. Der ACE2-Rezeptor ist auch in intestinalen Enterozyten sehr hoch exprimiert und virale Partikel konnten auch in intestinalen Zellen nachgewiesen werden. Der Gastrointestinaltrakt ist dabei einer der wichtigsten extrapulmonalen Manifestationen der COVID-19-Erkrankung. Dabei kann eine Schädigung des Gastrointestinaltraktes durch direkte zytotoxische Veränderungen, Dysregulation des Renin-Angiotensin-Aldosteron-Systems sowie auch Malabsorption entstehen. Schließlich kann auch ein direkter endothelialer Zellschaden und eine Mikroangiopathie eintreten.
Zusammenfassend wurde in dieser ersten allgemeinen wissenschaftlichen Sitzung in der deutschen Pathologie zur COVID-19-Pandemie deutlich, dass die PathologInnen in Deutschland, Österreich und der Schweiz sehr schnell auf die Pandemie reagiert haben und an zahlreichen Stellen ein Autopsieprogramm aufgebaut werden konnte. Diese Autopsien haben die Identifizierung von zahlreichen interessanten neuen Daten ermöglicht, die jetzt die Therapie von Patienten mit Covid-19-Infektion beeinflussen. Es wurde gezeigt, dass spezifische Grunderkrankungen häufig mit einem schweren Verlauf der Erkrankung assoziiert sind, die meisten Patienten aber an und nicht mit der Infektion versterben. 
Im inzwischen angelaufenen großen deutschlandweiten Forschungsprojekt DEFEAT PANDEMIcs ist der Aufbau eines deutschlandweiten Obduktionsnetzwerks für den Pandemiefall geplant, um systematisch Daten, Materialien und Erkenntnisse möglichst vollständig, umfassend und zeitnah zu erfassen. Über das Netzwerk werden die Generierung und Verbreitung von Evidenz zum Umgang mit Epidemien und zur Unterstützung eines Krisenmanagements durch die Erarbeitung standardisierter Vorgehensweisen erreicht. DEFEAT PANDEMIcs liefert so einen wertvollen Beitrag zur Bewältigung der aktuellen COVID-19 Pandemie sowie zur schnellen Reaktion auf künftige Pandemien.

\section{Korrespondenzadresse}

\section{Prof. Dr. P. Boor}

Institut für Pathologie \& Medizinische Klinik II (Nephrologie), Sektion Translationale Nephropathologie, Universitätsklinikum der RWTH Aachen

Pauwelsstr. 30, 52074 Aachen, Deutschland pboor@ukaachen.de

\section{Prof. Dr. A. Hartmann}

Institut für Pathologie, Universitätsklinikum Erlangen

Krankenhausstr. 8-10, 91054 Erlangen,

Deutschland

Arndt.Hartmann@uk-erlangen.de

\section{Einhaltung ethischer Richtlinien}

Interessenkonflikt. P. Boor und A. Hartmann geben an, dass kein Interessenkonflikt besteht.

Für diesen Beitrag wurden von den Autoren keine Studien an Menschen oder Tieren durchgeführt. Für die aufgeführten Studien gelten die jeweils dort angegebenen ethischen Richtlinien.

The supplement containing this article is not sponsored by industry.

\section{Literatur}

1. von Stillfried S et al (2020) Autopsy registry can facilitate COVID-19 research. EMBO Mol Med 12(8):e12885

2. Ackermann M et al (2020) Pulmonary vascular endothelialitis, thrombosis, and angiogenesis in Covid-19. NEngl JMed 383(2):120-128

3. Varga Z et al (2020) Endothelial cell infection and endotheliitis in COVID-19. Lancet 395(10234):1417-1418 